\title{
BMJ Open Effect of repetitive transcranial magnetic stimulation (rTMS) for insomnia: a protocol for a systematic review
}

\author{
Yu He, ${ }^{1,2}$ Nianyi Sun, ${ }^{1,2}$ Zhiqiang Wang, ${ }^{1,2}$ Wenchen Zou ${ }^{1}$
}

To cite: He Y, Sun N, Wang Z, et al. Effect of repetitive transcranial magnetic stimulation (rTMS) for insomnia: a protocol for a systematic review. BMJ Open 2019;9:e029206. doi:10.1136/ bmjopen-2019-029206

- Prepublication history and additional material for this paper are available online. To view please visit the journal (http:// dx.doi.org/10.1136/bmjopen2019-029206).

Received 16 January 2019 Revised 3 June 2019 Accepted 25 June 2019
Check for updates

(c) Author(s) (or their employer(s)) 2019. Re-use permitted under CC BY-NC. No commercial re-use. See rights and permissions. Published by BMJ.

${ }^{1}$ Department of Rehabilitation, Shengjing Hospital of China Medical University, Shenyang, China

${ }^{2}$ Department of Physical Medicine and Rehabilitation, The Second Clinical College, China Medical University, Shenyang, China

Correspondence to

Dr Nianyi Sun;

sny1986@hotmail.com

\begin{abstract}
Introduction Repetitive transcranial magnetic stimulation (rTMS), a non-invasive brain stimulation approach, might be a promising technique in the management of insomnia. A systematic review of the available literature on this topic is warranted. The systematic review described in this protocol aims to investigate the efficacy of rTMS as a physical therapy in patients with insomnia.

Methods and analysis This protocol was developed in accordance with the Preferred Reporting Items for Systematic Review and Meta-Analysis Protocols. We will retrieve relevant literatures across the following electronic bibliographic databases: CENTRAL, PubMed, EMBASE, PsycINFO, CINAHL, PEDro, CBM, CNKI, WANFANG and VIP. A manual search of the reference lists of all relevant articles will be performed for any additional studies. We will include randomised controlled trials published in English and Chinese examining efficacy of rTMS on patients with insomnia. Two reviewers will independently complete the article selection, data extraction and rating. PEDro scale will be used to assess the methodological quality of the included studies. Narrative and quantitative synthesis will be done accordingly.

Ethics and dissemination Ethical approval will not be required for this review. The results of this review will be disseminated in a peer-review journal.
\end{abstract}

PROSPERO registration number CRD42018115033.

\section{INTRODUCTION}

Insomnia disorder is a patient-reported problem characterised by difficulty initiating sleep, difficulty maintaining sleep, early morning awakening or non-restorative or poor sleep quality. ${ }^{12}$ It is a common sleep disorder, recent data show that the population prevalence of insomnia is estimated to be $5 \%-16 \% .^{3-7}$ Compared with good sleepers, individuals with the disorder often experience clinically significant distress or daytime dysfunction such as fatigue, poor cognitive function, emotional disturbance and poor performance, ${ }^{8}$ have higher risk for many physical health problems (cardiovascular diseases, ${ }^{9}$ neurological disorders, ${ }^{10}$ obesity, ${ }^{11}$ diabetes ${ }^{12} 13$ and so on) as well as mental health problems (such as depression, anxiety, suicide and substance use), ${ }^{14-18}$ and

\section{Strengths and limitations of this study}

- Well-recognised approach for conducting and reporting of systematic reviews will be followed (Cochrane handbook).

- To our knowledge, this study will be the first to explore the efficacy of repetitive transcranial magnetic stimulation (rTMS) as a physical therapy in patients with insomnia.

- In the study, both primary and comorbid insomnia will be included.

- This work provides an overview of the current situation in this territory and will potentially be helpful for future evidence-based research to refine rTMS.

- The scarcity of randomised controlled trials and the methodological quality of studies may be the main limitations of the study.

have increased risk of errors and accidents, increased work absenteeism rates and healthcare use. ${ }^{19}{ }^{20}$ Insomnia has a large negative impact on function, health and quality of life, resulting in a major public health burden. ${ }^{21}$

General approaches in the management of insomnia include the evaluation and treatment of contributing medical or psychiatric comorbidities, and specific treatment strategies fall into two major categories: pharmacological treatments and cognitive behavioural therapy (CBT).$^{22}$ Due to potential adverse effects and limited supporting data of sleep-promoting medication, clinical practices and guidelines recommended CBT as the first-line treatment for insomnia, and pharmacotherapy may be used on a short-term basis if CBT is either inaccessible or ineffective. ${ }^{23-26}$ However, CBT is rarely used in routine clinical practice, possible reasons include time consumption and lack of adequately trained providers, other barriers may be related to patient awareness and economic issues. ${ }^{24} 2728$ Given the limitations of both pharmacological therapy and CBT for insomnia, treating insomnia can be challenging. Other than improving access to CBT, many complementary therapies have 
been used to improve sleep, and repetitive transcranial magnetic stimulation (rTMS), a non-invasive brain stimulation approach, might be a promising technique.

TMS is a non-invasive method of stimulating the brain through the intact skull and scalp with little or no pain at the surface. ${ }^{29-31}$ In TMS, a magnetic field is generated with a magnetic coil placed above the scalp, which efficiently passes through the skull and induces electrical currents in the underlying brain tissue beneath the coil, and the induced electrical stimulus activates neural elements in the cortex or subcortical white matter. ${ }^{31}{ }^{32} \mathrm{~A}$ single TMS pulse lasts only $\sim 0.2-0.3 \mathrm{~ms}$, which induces neuronal effects lasting only a fraction of a second, thus producing short responses. By contrast, longer rTMS pulse sequences, such as conventional $1 \mathrm{~Hz}$ or $20 \mathrm{~Hz}$ rTMS which have usually been delivered in trains, can cause prolonged neuroplastic changes that inhibit or excite the brain's activity. ${ }^{30-32}$ rTMS has been reported to have therapeutic utility in many neurological and psychiatric conditions, such as stroke, ${ }^{33}$ Parkinson's disease, ${ }^{34}$ dystonia, ${ }^{35}{ }^{36}$ depression ${ }^{37}$ and schizophrenia. ${ }^{38}$

Among several models of insomnia, hyperarousal is identified as a final common pathway of the pathophysiology ${ }^{39-41}$ In addition, studies using TMS to assess the trait of insomnia found that patients with insomia are characterised by disturbed intracortical excitability. ${ }^{42} 43$ Reduction of overexcited state in patients with insomia can effectively improve their sleep. ${ }^{44}$ The potential mechanisms of rTMS in improving sleep in patients with insomnia might involve inhibiting the hyperarousal state of the cerebral cortex, affecting metabolic activity and sleep-related hormones, and promoting hippocampal neurogenesis. ${ }^{3945} 46$ In the recent years, there is an increasing interest in application of rTMS in the treatment of patients with insomia, and it seems that rTMS might have the potential to improve sleep ${ }^{454748}$ but negative results were also reported. ${ }^{49}$ Until now, there is no systematic review about rTMS for insomnia. We seek to perform a systematic review of randomised controlled trials (RCTs) to evaluate the efficacy of rTMS as a physical therapy in patients with insomnia.

\section{METHODS}

This protocol was registered prospectively in PROSPERO, it was developed in accordance with the Preferred Reporting Items for Systematic Review and Meta-Analysis Protocols. ${ }^{50}$

\section{Research question}

The PICO ( $\mathrm{P}=$ Patients, $\mathrm{I}=$ Intervention, $\mathrm{C}=$ Comparison, $\mathrm{O}=$ Outcomes) method will be used to define the major components of this systematic review:

- P: adults ( $>18$ years of age) of any gender and ethnic background with insomnia diagnosed by standard criteria such as Diagnostic and Statistical Manual of Mental Disorders (Fourth Edition, ${ }^{52}$ Fifth Edition ${ }^{1}$ ), International Classification of Sleep Disorders
(Second Edition, ${ }^{53}$ Third Edition ${ }^{2}$ ), International Classification of Diseases (Tenth Edition),${ }^{54}$ Chinese Classification and Diagnostic Criteria of Mental Disorders (Third Edition). ${ }^{55}$ In the study, both primary and comorbid insomnia will be included.

- I: the intervention used in the experimental group was rTMS alone or in combination with other therapy. The intensity of stimulation, frequency, target area, localisation method, dose and duration are not limited.

- C: comparison with placebo or sham control, other treatment, no intervention or no rTMS therapy under the same treatment programme.

- O: primary outcomes and secondary outcomes.

Primary outcomes:

- Polysomnography: sleep parameters such as total sleep time (TST), sleep efficiency (SE), wake after sleep onset (WASO), sleep onset latency (SO, rapid-eye movement (REM) latency, total arousal index, percentage of TST spent in each sleep stage (N1, N2, N3, REM), apnoea-hypopnoea index, mean arterial oxygen saturation $\left(\mathrm{SaO}_{2}\right)$, minimum $\mathrm{SaO}_{2}$ and periodic limb movements index.

- Pittsburgh Sleep Quality Index.

Secondary outcomes:

- Other sleep-related scales: Insomnia Severity Index, Athens Insomnia Scale.

- Sleep parameters measured by actigraphy: activity mean, sleep duration, sleep onset latency, SE, WASO, daytime inactivity.

- Adverse events: number and type of self-reported and measured adverse events during the study period.

Thus, this systematic review aims to answer the following question: Is rTMS effective in improving quality of sleep compared with other forms of treatment or no intervention?

\section{Eligibility criteria}

In addition to the PICO components mentioned above, articles will be included for review if they were:

- Published in the English or Chinese language.

- RCTs.

\section{Exclusion criteria}

Articles will be excluded if:

- Insufficient data for the establishment of results were reported (eg, deficiency of the number of participants, the means and the SD or other relevant statistics regarding a continuous outcome).

- They were reported in books, conference proceedings or dissertations.

\section{Search strategy}

We will retrieve relevant literatures across the following electronic bibliographic databases: Cochrane Central Register of Controlled Trials (CENTRAL), PubMed, EMBASE, PsycINFO, CINAHL, Physiotherapy Evidence Database (PEDro), the Chinese Biomedical Literature 
Database (CBM), China National Knowledge Infrastructure (CNKI), WANFANG database, and the Chinese Scientific Journal Database (VIP). We will develop the search strategy for MEDLINE (via Ovid) (see supplementary material online supplementary appendix 1 . Search Strategy Example) and adapt this strategy for the other databases except for PEDro in which the term 'rTMS' and 'insomnia' will be used for simple search. The concepts of 'subjects' and 'intervention' will be combined with the 'AND' operator. Subjects are defined as individuals with insomnia, and intervention is defined as rTMS. For each concept, we will combine synonyms and Medical Subject Headings terms with the 'OR' operator. In addition to database searching, a manual search of the reference lists of all relevant articles will be performed for any additional studies.

\section{Selection of studies}

Duplicate articles generated by different databases will be removed using an electronic reference management tool Endnote X7 software. The title, abstract and keywords of every retrieved articles will be scanned independently by two authors (NS and $\mathrm{YH}$ ) with predetermined eligibility criteria, in order to eliminate the irrelevant studies and determine which studies require further assessment. If there is any doubt regarding these criteria from scanning the titles and abstracts, the full article will be retrieved for clarification. Later, the full text of the remaining articles after preliminary screening will be reviewed in detail, and the criteria will be reapplied to these studies to determine the eligible ones. Discrepancies will be resolved by discussion (or with consultation of a third author); decisions will be arrived at by the authors in consensus.

\section{Data extraction}

Two authors (NS and $\mathrm{YH}$ ) will independently extract relevant population and intervention characteristics from the included studies. The data will include the following items:

- General information: first author, title, journal, year of publication, country, setting, aim, ethical approval, register number and funding source.

- Trial characteristics: design, method of randomisation, allocation concealment, blinding (subjects, therapists, and assessors).

- Intervention(s): intervention(s) (stimulus frequency, number of TMS pulses delivered per session, target area, localisation method, number of sessions in a course of treatment), comparison intervention(s) (type, frequency, dose and duration).

- Participants: participant demographics, number of participants, inclusion/exclusion criteria, comorbidity, assessment of compliance and withdrawals.

- Outcomes: results, adverse events, duration of follow-up, intention-to-treat (ITT) analysis.

\section{Methodological quality assessment}

This review will use The Physiotherapy Evidence Database (PEDro) scale as methodological criteria. ${ }^{56}$ The PEDro scale is an instrument for the methodological quality assessment of physiotherapy-based studies. It had been demonstrated that PEDro scale possesses moderate inter-rater reliability for assessing methodological quality of clinical trials. ${ }^{56} 57$ This scale has 11 criteria, with scores range from 0 to 10 (the 1st item-specified eligibility criteria-is not included in the total score), and a higher score indicates that the trial has a better methodological quality $(9-10=$ excellent, $6-8=$ good, $4-5=$ =fair, and $<4=$ poor). Responses to the 2 nd to 11th item are summed to create a total score, which include randomisation, allocation concealment, baseline comparability, blinding (subjects, therapists, and assessors), follow-up of subjects (at least 85\%), ITT analysis, betweengroup statistical comparisons, and reporting point measures and measures of variability. ${ }^{56}$ The scores of each selected study will be determined by two authors (NS and $\mathrm{YH}$ ) independently based on these criteria. Possible disagreement will be resolved by consensus (discussion or with consultation of a third author).

\section{Data analysis and synthesis}

The Cochrane Review Manager V.5 software will be used for meta-analysis. In our study, meta-analysis concerning effects of rTMS will be conducted if two or more studies used the same outcome measure or measured similar constructs.

For continuous data, standardised mean differences as well as $95 \%$ CI will be computed. For dichotomous data, OR as well as $95 \%$ CI will be computed. We will employ the $\chi^{2}$ test to analyse the heterogeneity, a $p$ value $\leq 0.1$ will be considered statistically significant. ${ }^{58}$ Heterogeneity between studies will be calculated using the $\mathrm{I}^{2}$ statistic, which indicates the proportion of variation across studies. ${ }^{59}$ If $\mathrm{p}>0.1, \mathrm{I}^{2}<50 \%$, fixed effects model will be used; Substantial heterogeneity is considered If $\mathrm{p}>0.1, \mathrm{I}^{2} \geq 50 \%$, and random effects model will be used in this case; If $p \leq 0.1$, statistical heterogeneity is considered, subgroup analysis or a narrative description will be conducted depending on the available data. ${ }^{58}$

If data permitted, we will carry out subgroup analyses on the following factors to assess whether the treatment effects are different in different subgroups: diagnostic subtypes (primary insomnia versus comorbid insomnia), age groups, site of stimulation, nature of the comparative treatment. 58

When sufficient data are available, we will carry out sensitivity analyses on the following factors to assess the impact of study quality: concealed allocation, outcomes assessor blinding, drop outs, ITT analysis. ${ }^{58}$

The results of meta-analysis will be presented as forest plots. If $\geq 10$ studies are included in the meta-analysis, potential publication bias will be assessed by graphing a funnel plot. ${ }^{58}$

The quality of the evidence in this review will be assessed by GRADEpro V.3 software and rated into high, moderate, low or very low, four levels according to the 
Grading of Recommendations Assessment, Development and Evaluation system. ${ }^{60}$

\section{Patient and public involvement}

Not applicable. This systematic review protocol does not directly involve patients and the general public. Data will be collected from published articles retrieved from main databases and manual search.

\section{ETHICS AND DISSEMINATION}

Ethical approval will not be required for the performance of this review. Results of this research will be disseminated in a peer-review journal. These results will potentially be helpful for improving the therapeutic strategy of patients with insomnia.

Contributors $\mathrm{YH}, \mathrm{ZW}, \mathrm{WZ}$ and NS contributed to the conception and design of the study. YH and NS drafted the protocol. NS registered the protocol review in the PROSPERO database, revised the protocol. NS and YH will carry out the data search, study selection, data extraction, methodological quality assessment, data analysis, drafting and revising of the final review. ZW provided critical insights and will help with the development of final review. WZ designed the search strategy, helped with the data collection and will help with the final review. All authors have read and approved the final submitted version of this protocol. NS is the guarantor of the review.

Funding The authors have not declared a specific grant for this research from any funding agency in the public, commercial or not-for-profit sectors.

Competing interests None declared.

Patient consent for publication Obtained.

Provenance and peer review Not commissioned; externally peer reviewed.

Open access This is an open access article distributed in accordance with the Creative Commons Attribution Non Commercial (CC BY-NC 4.0) license, which permits others to distribute, remix, adapt, build upon this work non-commercially, and license their derivative works on different terms, provided the original work is properly cited, appropriate credit is given, any changes made indicated, and the use is non-commercial. See: http://creativecommons.org/licenses/by-nc/4.0/.

\section{REFERENCES}

1. American Psychiatric Association. Diagnostic and Statistical Manual of Mental Disorders, Fifth Edition (DSM-V). Arlington, VA: American Psychiatric Association, 2013.

2. American Academy of Sleep Medicine. International Classification of Sleep Disorders, Third Edition (ICSD-III). Darien, IL: American Academy of Sleep Medicine, 2014.

3. Chung KF, Yeung WF, Ho FY, et al. Cross-cultural and comparative epidemiology of insomnia: the Diagnostic and statistical manual (DSM), International classification of diseases (ICD) and International classification of sleep disorders (ICSD). Sleep Med 2015;16:477-82.

4. Hillman DR, Lack LC. Public health implications of sleep loss: the community burden. Med J Aust 2013;199:7-10.

5. Pallesen S, Sivertsen B, Nordhus IH, et al. A 10-year trend of insomnia prevalence in the adult Norwegian population. Sleep Med 2014;15:173-9.

6. Morin CM, LeBlanc M, Bélanger L, et al. Prevalence of insomnia and its treatment in Canada. Can J Psychiatry 2011;56:540-8.

7. Cao XL, Wang SB, Zhong BL, et al. The prevalence of insomnia in the general population in China: A meta-analysis. PLoS One 2017;12:e0170772.

8. Buysse DJ. Insomnia. JAMA 2013;309:706-16.

9. Javaheri S, Redline S. Insomnia and risk of cardiovascular disease. Chest 2017;152:435-44.

10. Ju YS, Videnovic A, Vaughn BV. Comorbid sleep disturbances in neurologic disorders. Continuum 2017;23:1117-31.

11. Chan WS, Levsen MP, McCrae CS. A meta-analysis of associations between obesity and insomnia diagnosis and symptoms. Sleep Med Rev 2018;40:170-82.
12. Lin CL, Chien WC, Chung $\mathrm{CH}$, et al. Risk of type 2 diabetes in patients with insomnia: A population-based historical cohort study. Diabetes Metab Res Rev 2018;34:e2930.

13. Grandner MA, Seixas A, Shetty $S$, et al. Sleep Duration and Diabetes Risk: Population Trends and Potential Mechanisms. Curr Diab Rep 2016;16:106.

14. Chung KH, Li CY, Kuo SY, et al. Risk of psychiatric disorders in patients with chronic insomnia and sedative-hypnotic prescription: a nationwide population-based follow-up study. J Clin Sleep Med 2015;11:543-51.

15. Neckelmann D, Mykletun A, Dahl AA. Chronic insomnia as a risk factor for developing anxiety and depression. Sleep 2007;30:873-80.

16. Taylor DJ, Bramoweth AD, Grieser EA, et al. Epidemiology of insomnia in college students: relationship with mental health, quality of life, and substance use difficulties. Behav Ther 2013;44:339-48.

17. Lin HT, Lai CH, Perng HJ, et al. Insomnia as an independent predictor of suicide attempts: a nationwide population-based retrospective cohort study. BMC Psychiatry 2018;18:117.

18. Woznica AA, Carney CE, Kuo JR, et al. The insomnia and suicide link: toward an enhanced understanding of this relationship. Sleep Med Rev 2015;22:37-46.

19. Daley M, Morin CM, LeBlanc M, et al. Insomnia and its relationship to health-care utilization, work absenteeism, productivity and accidents. Sleep Med 2009;10:427-38.

20. Shahly V, Berglund PA, Coulouvrat C, et al. The associations of insomnia with costly workplace accidents and errors: results from the America Insomnia Survey. Arch Gen Psychiatry 2012;69:1054-63.

21. Alonso J, Vilagut G, Chatterji S, et al. Including information about co-morbidity in estimates of disease burden: results from the World Health Organization World Mental Health Surveys. Psychol Med 2011;41:873-86.

22. Buysse DJ, Rush AJ, Reynolds CF. Clinical management of insomnia disorder. JAMA 2017;318:1973-4.

23. Winkelman JW. Clinical practice. Insomnia Disorder. N Engl J Med 2015;373:1437-44

24. Kay-Stacey M, Attarian $\mathrm{H}$. Advances in the management of chronic insomnia. BMJ 2016;354:i2123.

25. Qaseem A, Kansagara D, Forciea MA, et al. Management of chronic insomnia disorder in adults: a clinical practice guideline from the American College of Physicians. Ann Intern Med 2016;165:125-33.

26. Riemann D, Baglioni $C$, Bassetti $C$, et al. European guideline for the diagnosis and treatment of insomnia. J Sleep Res 2017;26:675-700.

27. Medalie L, Cifu AS. Management of Chronic Insomnia Disorder in Adults. JAMA 2017;317:762-3.

28. Morin CM. Issues and challenges in implementing clinical practice guideline for the management of chronic insomnia. J Sleep Res 2017;26:673-4.

29. Barker AT, Jalinous R, Freeston IL. Non-invasive magnetic stimulation of human motor cortex. Lancet 1985;1:1106-7.

30. Hallett M. Transcranial magnetic stimulation and the human brain. Nature 2000;406:147-50.

31. Ridding MC, Rothwell JC. Is there a future for therapeutic use of transcranial magnetic stimulation? Nat Rev Neurosci 2007;8:559-67.

32. Diana M, Raij T, Melis M, et al. Rehabilitating the addicted brain with transcranial magnetic stimulation. Nat Rev Neurosci 2017;18:685-93.

33. Hao Z, Wang D, Zeng Y, et al. Repetitive transcranial magnetic stimulation for improving function after stroke. Cochrane Database Syst Rev 2013;5:CD008862.

34. Chung CL, Mak MK. Effect of repetitive transcranial magnetic stimulation on physical function and motor signs in parkinson's disease: a systematic review and meta-analysis. Brain Stimul 2016:9:475-87.

35. Kimberley TJ, Borich MR, Arora S, et al. Multiple sessions of lowfrequency repetitive transcranial magnetic stimulation in focal hand dystonia: clinical and physiological effects. Restor Neurol Neurosci 2013;31:533-42.

36. Huang $\mathrm{YZ}$, Edwards MJ, Bhatia KP, et al. One-Hz repetitive transcranial magnetic stimulation of the premotor cortex alters reciprocal inhibition in DYT1 dystonia. Mov Disord 2004;19:54-9.

37. Berlim MT, Van den Eynde F, Jeff Daskalakis Z. Clinically meaningful efficacy and acceptability of low-frequency repetitive transcranial magnetic stimulation (rTMS) for treating primary major depression: a meta-analysis of randomized, double-blind and sham-controlled trials. Neuropsychopharmacology 2013;38:543-51.

38. Hovington CL, McGirr A, Lepage M, et al. Repetitive transcranial magnetic stimulation (rTMS) for treating major depression and schizophrenia: a systematic review of recent meta-analyses. Ann Med 2013;45:308-21.

39. Riemann D, Spiegelhalder K, Feige B, et al. The hyperarousal model of insomnia: a review of the concept and its evidence. Sleep Med Rev 2010;14:19-31. 
40. Riemann D, Nissen C, Palagini L, et al. The neurobiology, investigation, and treatment of chronic insomnia. Lancet Neurol 2015;14:547-58.

41. Levenson JC, Kay DB, Buysse DJ. The pathophysiology of insomnia. Chest 2015;147:1179-92.

42. van der Werf YD, Altena E, van Dijk KD, et al. Is disturbed intracortical excitability a stable trait of chronic insomnia? A study using transcranial magnetic stimulation before and after multimodal sleep therapy. Biol Psychiatry 2010;68:950-5.

43. Lanza G, Cantone M, Lanuzza B, et al. Distinctive patterns of cortical excitability to transcranial magnetic stimulation in obstructive sleep apnea syndrome, restless legs syndrome, insomnia, and sleep deprivation. Sleep Med Rev 2015;19:39-50.

44. Cortoos A, Verstraeten E, Cluydts R. Neurophysiological aspects of primary insomnia: implications for its treatment. Sleep Med Rev 2006;10:255-66.

45. Jiang CG, Zhang T, Yue FG, et al. Efficacy of repetitive transcranial magnetic stimulation in the treatment of patients with chronic primary insomnia. Cell Biochem Biophys 2013;67:169-73.

46. Strafella AP, Paus T, Fraraccio M, et al. Striatal dopamine release induced by repetitive transcranial magnetic stimulation of the human motor cortex. Brain 2003;126(Pt 12):2609-15.

47. Park EJ, Lee SJ, Koh DY, et al. Repetitive transcranial magnetic stimulation to treat depression and insomnia with chronic low back pain. Korean J Pain 2014;27:285-9.

48. Huang Z, Li Y, Bianchi MT, et al. Repetitive transcranial magnetic stimulation of the right parietal cortex for comorbid generalized anxiety disorder and insomnia: A randomized, double-blind, shamcontrolled pilot study. Brain Stimul 2018;11:1103-9.

49. Arias P, Vivas J, Grieve KL, et al. Double-blind, randomized, placebo controlled trial on the effect of 10 days low-frequency rTMS over the vertex on sleep in Parkinson's disease. Sleep Med 2010;11:759-65.
50. Moher D, Shamseer L, Clarke M, et al. Preferred reporting items for systematic review and meta-analysis protocols (PRISMA-P) 2015 statement. Syst Rev 2015;4:1.

51. Shamseer L, Moher D, Clarke M, et al. Preferred reporting items for systematic review and meta-analysis protocols (PRISMA-P) 2015 elaboration and explanation. BMJ 2015;350:g7647.

52. American Psychiatric Association. Diagnostic and Statistical Manual of Mental Disorders, Forth Edition (DSM-IV). Washington, DC: American Psychiatric Association, 1994.

53. American Academy of Sleep Medicine. International Classification of Sleep Disorders, Second Edition (ICSD-II). Westchester, IL: American Academy of Sleep Medicine, 2005.

54. World Health Organization. International Statistical Classification of Diseases and Related Health Problems, Tenth Revision. Geneva: World Health Organization, 1992.

55. Psychosis Branch of Chinese Medical Association. Chinese Classification and Diagnostic Criteria of Mental Disorders, Third Edition (CCMD-3). Jinan: Shandong Science and Technology Publishing, 2001

56. Maher CG, Sherrington C, Herbert RD, et al. Reliability of the PEDro scale for rating quality of randomized controlled trials. Phys Ther 2003;83:713-21.

57. de Morton NA. The PEDro scale is a valid measure of the methodological quality of clinical trials: a demographic study. Aust $J$ Physiother 2009;55:129-33.

58. Higgins JPT, Green S. Cochrane handbook for systematic reviews of interventions, version 5.1.0: The Cochrane Collaboration, 2011.

59. Higgins JP, Thompson SG, Deeks JJ, et al. Measuring inconsistency in meta-analyses. BMJ 2003;327:557-60.

60. Guyatt GH, Oxman AD, Vist GE, et al. GRADE: an emerging consensus on rating quality of evidence and strength of recommendations. BMJ 2008;336:924-6. 